\title{
MARKETING DE SERVIÇOS: RETROSPECTIVA E TENDÊNCIAS
}

\author{
Angela da Rocha \\ Professora da Coppead/UFRJ \\ E-mail: angela@coppead.ufrj.br \\ Jorge Ferreira da Silva \\ Professor do IAG/PUC-Rio \\ E-mail: shopshop@iag.puc-rio.br
}

\section{INTRODUÇÃO}

O interesse pelo marketing de serviços, particularmente nos últimos 20 anos, encontra-se ligado à importância econômica dos serviços nas economias pós-industriais. Mais ainda, à medida que se reduzem as possibilidades de diferenciação de produtos, devido ao amadurecimento dos mercados, as empresas são levadas a desenvolver vantagens competitivas centradas na oferta ampliada de serviços.

Ainda que a atenção dada aos serviços seja relativamente recente na literatura de marketing, estudos pioneiros nessa área datam da década de 1960 (Cronin, 2003). $O$ interesse inicial estava centrado na distribuição de serviços e na aplicação do marketing a esse setor.

Kotler (1995) atribui a Shostack (1977), em artigo pioneiro publicado no Journal of Marketing, intitulado "Breaking free from product marketing", a responsabilidade de "alterar o curso de nosso pensamento sobre marketing de serviços, se não do próprio marketing em geral" (p. 13). Para Kotler, foi a partir desse artigo, que questionava a aplicabilidade do marketing de produtos aos serviços, que pesquisadores e estudiosos da área desenvolveram os fundamentos do que viria a ser uma teoria de marketing aplicada a serviços. Esse ponto de vista é apoiado por Brown, Fisk e Bitner (1994), em sua ampla revisão do desenvolvimento do pensamento de marketing em serviços, que consideram o artigo de Shostack como um demarcador de fronteiras. Sua publicação gerou toda uma seqüência de publicações em que se discutiam a natureza dos serviços, suas características e a aplicabilidade dos conceitos tradicionais de marketing ao marketing de serviços.

Entre os pioneiros no desenvolvimento teórico do marketing de serviços podem ser citados Grönroos (1983, 1984, 1995), Normann $(1977,1993)$ e Lovelock (1983, 1984). Grönroos e Normann são dos muitos estudiosos escandinavos do tema, que também teve repercussão nos Estados Unidos, no Reino Unido e na França, países onde rapidamente se formaram grupos integrados de pesquisa. Um dos artigos de Grönroos (1984), reconhecidamente um dos autores que mais contribuíram para o desenvolvimento desse campo de estudos, foi incluído neste número da "RAE-Clássicos". Trata-se de um trabalho pioneiro, em que o autor desenvolve o primeiro modelo integrado de qualidade de serviços (Seth, Deshmukh e Vrat, 2005). É do pensamento de Grönroos que se difundiram os conceitos iniciais de qualidade percebida dos serviços, e suas diferentes vertentes: a qualidade técnica e a qualidade funcional.

No plano empresarial, a difusão da importância da qualidade de serviços nas interações com o cliente foi fortemente influenciada por Carlzon (1990), que aplicou com sucesso os "momentos da verdade" de Normann à empresa de aviação civil SAS. A publicação do livro de Carlzon serviu efetivamente como mecanismo propagador do marketing de serviços.

Outro divisor de águas na evolução do novo campo de estudos foram os trabalhos publicados por Parasuraman, Zeithaml e Berry (1985, 1988), e Parasuraman, Berry e Zeithaml (1991). Esses autores publicaram em 1985 um artigo que viria a ter grande influência no desenvolvimento das pesquisas posteriores, intitulado "A conceptual model of service quality and its implications for future research", que também foi escolhido para compor essa edição da "RAE-Clássicos". Seu impacto foi tão grande que as investigações sobre qualidade de serviços assumiram, desde então, papel central nos estudos de 
marketing de serviços. No artigo, os autores apresentam o modelo de gaps ou hiatos de qualidade, que evoluiu para a conhecida escala Servqual, um instrumento de medida da qualidade percebida de serviços (Parasuraman, Zeithaml e Berry, 1988). Trabalhos posteriores dos autores buscaram o refinamento da escala (Parasuraman, Berry e Zeithaml, 1991; Parasuraman, Zeithaml e Berry, 1994). Além disso, inúmeros testes e replicações foram realizados em diversos países e subsetores de serviços, adaptando e testando a escala Servqual (Asubonteng, McCleary e Swan, 1996).

A importância dos estudos da qualidade de serviços pode ser atestada por recente revisão crítica dos modelos de qualidade de serviços, realizada por Seth, Deshmukh e Vrat (2005), em que são examinados 19 modelos, apresentados entre 1984 e 2003. Os autores concluem que "não parece existir uma definição conceitual nem um modelo de qualidade de serviços amplamente aceitos, e nem definições operacionais geralmente aceitas do modo de medir qualidade de serviços" (p. 933-934), ainda que haja consistência entre a maioria dos modelos em comparar expectativas com experiências de serviços. Entre os vários modelos examinados, os autores observaram que a maioria era derivada do Servqual, confirmando o fato de essa escala apresentar ampla aceitação e difusão, tanto no meio acadêmico quanto no meio empresarial.

A crescente importância do marketing e da gestão de serviços pode ser atestada pela existência de revistas científicas dedicadas especificamente ao tema, tais como Journal of Services Marketing, Services Marketing Quarterly, The Service Industries Journal e International Journal of Service Industry Management. Também é grande o número de congressos e outras reuniões científicas específicas, além de centros de pesquisa, dedicados exclusivamente ao tema (Grove, Fisk e John, 2003).

Brown, Fisk e Bitner (1994, p. 21) afirmam que nenhum outro campo dentro da disciplina de Marketing, exceto o de comportamento do consumidor, foi objeto de tanta "paixão e determinação" em seu desenvolvimento. Os autores salientam três fatores que explicariam o crescimento da disciplina, possibilitando a fertilização cruzada: (i) a forte interação entre a academia e as empresas; (ii) o caráter interdisciplinar da literatura, com o envolvimento das áreas de operações e recursos humanos; (iii) o fato de a literatura sobre marketing de serviços haver germinado em diversos países, tendo, desde o início, caráter internacional. Martin (1999) qualifica o desenvolvimento teórico desse campo como "surpreendente e acelerado", reconhecendo ainda a interdisciplinaridade como carac- terística, enquanto Laing et al. (2002) sugerem como um dos fatores que contribuíram para a expansão do conhecimento o próprio desenvolvimento dos serviços, especialmente a desregulamentação e a mudança tecnológica. Para esses autores, o impacto da Internet no setor de serviços ocorreu primeiramente nos serviços que não requerem interação interpessoal, em particular nos serviços financeiros e de viagens. Em seguida avançou gradativamente para aqueles serviços com alto componente de interação interpessoal, como é o caso dos serviços profissionais.

\section{TRABALHOS REALIZADOS NO BRASIL}

O conhecimento desenvolvido no país sobre marketing de serviços é já bastante expressivo, ainda que concentrado em algumas áreas específicas. Uma análise dos trabalhos apresentados em sete reuniões anuais da Anpad, entre 1999 e 2005, permitiu identificar um total de 50 trabalhos, inseridos em quatro grandes linhas de pesquisa: cenários de serviços, processos de escolha de serviços, comportamento pós-compra de serviços e estratégia de marketing de serviços. No entanto, poucos trabalhos foram publicados nas principais revistas científicas da área de Administração no Brasil, salientando-se os de Moori, Marcondes e Ávila (2002), Marchetti e Prado (2004), Larán e Espinoza (2004), Slongo e Müssnich (2005), e Brei e Rossi (2005). Entre aqueles publicados, a maioria foi previamente apresentada no EnAnpad, sugerindo que a análise dos artigos apresentados nesse evento científico pode proporcionar uma idéia acurada do estado da arte no Brasil.

Foram considerados somente aqueles trabalhos que utilizaram arcabouço teórico proveniente das teorias de marketing de serviços. Não foram considerados trabalhos cujo lócus de pesquisa se encontrava em empresas de serviços, mas cuja inserção conceitual e teórica era distinta. Nessa linha, diversos trabalhos sobre marketing de relacionamento no setor de serviços não foram incluídos na análise, nem trabalhos que versavam puramente sobre comportamento de compra na Internet ou no varejo. Finalmente, deve-se salientar que diversos trabalhos sobre gerência de operações de serviços encontram-se bastante próximos daqueles desenvolvidos em marketing de serviços, mas não foram levados em consideração para esta breve avaliação do estado do conhecimento no Brasil.

O Quadro 1 apresenta uma relação dos principais construtos e variáveis investigados nos trabalhos examinados. 
Quadro 1 - Principais construtos e variáveis em trabalhos de marketing de serviços nos EnAnpad (1999-2005)

CONSTRUTOS/VARIÁVEIS

Cenários de serviços

Elementos tangíveis

Pessoal de contato

Iluminação

Música

\section{Processo de escolha de serviços}

Risco percebido

Conjunto de consideração

Fidelidade à marca

Atributos de escolha

Comportamento pós-compra de serviços

Qualidade

Valor percebido

Satisfação

Lealdade, fidelidade

Confiança

Intenção de recompra

Emoção, resposta afetiva

Expectativa

Justiça

Custos de mudança

Tolerância

Recuperação do serviço

Imagem

Importância de serviços

Estratégia de marketing de serviços

Estrutura

Comprometimento da alta direção

Sistemas de avaliação e recompensa

Desempenho

\section{TRABALHOS}

Andrade (2004).

Andrade (2004); Carvalho, Faria e Carvalho (2002); Carvalho e Vergara (2000).

Carvalho e Motta (2000); Carvalho, Lima e Motta (2003).

Carvalho, Hemais e Motta (2003).

Brasil (2002); Fonseca, Neto, Cordeiro e Gouveia (2002); Neto (2003).

Neto, Gouveia, Cordeiro, Fonseca (2002); Neto (2003).

Neto, Gouveia, Cordeiro, Fonseca (2002).

Ghisi, Merlo e Nagano (2004).

Almeida e Botelho (2005); Carvalho e Leite (1999); Dutra, Oliveira e Gouveia (2002); Gonçalves Filho, Guerra e Moura (2003); Leite, Elias e Sunderman (2005); Moura (2005); Oliveira e Dutra (2002); Prado (2002); Rocha e Oliveira (2003); Rodrigues (2000b); Santos e Muniz (2004); Urdan e Huertas (2003); Vecchi e Luce (2001); Veiga, Gonçalves e Moura (2002). Almeida e Botelho (2005); Brei e Rossi (2002); Fonseca e Trez (2005); Gonçalves Filho, Guerra e Moura (2003); Moura (2005); Santos e Fernandes (2005).

Almeida e Botelho (2005); De Toni, Milan e Barazetti (2004); Farias e Santos (2000); Farias, Góis e Oliveira (2000); Gastal e Luce (2005); Gonçalves Filho, Guerra e Moura (2003); Leite, Elias e Sunderman (2005); Marchetti e Prado (2001); Moura (2005); Prado (2002); Révillion e Rossi (2000); Rodrigues (2000b); Rodrigues (2004); Rosa e Kamakura (2001); Rossi, Luce, Mazzon e Slongo (2004); Santos (2001); Santos e Fernandes (2005); Veiga, Gonçalves e Moura (2002); Zuñiga e Urdan (2000).

Brei e Rossi (2002); Cortimiglia, Macadar e Dinato (2003); Fonseca e Trez (2005); Gastal e Luce (2005); Gonçalves Filho, Guerra e Moura (2003); Leite, Elias e Sunderman (2005); Machado (2004); Moura (2005); Rodrigues (2004); Santos (2001); Santos e Fernandes (2005); Zuñiga e Urdan (2000).

Almeida e Botelho (2005); Brei e Rossi (2002); Brei (2003); Fonseca e Trez (2005); Santos (2001); Santos e Rossi (2002); Santos e Fernandes (2005).

Santos e Fernandes (2005).

Farias e Santos (2000); Prado (2002).

Gonçalves Filho, Guerra e Moura (2003); Leite e Carvalho (2000); Rocha e Oliveira (2003); Vecchi e Luce (2001).

Santos e Rossi (2002); Santos e Fernandes (2005).

Gastal e Luce (2005); Santos e Fernandes (2005).

Carvalho e Leite (1999); Rodrigues (2000a).

Cortimiglia, Macadar e Dinato (2003); Fonseca e Trez (2005); Santos e Fernandes (2005).

De Toni, Milan e Barazetti (2004); De Toni, Milan e Schuler (2005); Leite, Elias e Sunderman (2005).

Buss, Brei, Grinberg, Almeida e Freitas (2000).

Neto (2000).

Neto (2000).

Neto (2000).

Trez e Luce (2000); Trez e Luce (2001). 
Considerando-se as quatro linhas de pesquisa em que se realizaram desenvolvimentos teóricos e testes empíricos sobre marketing de serviços no Brasil, observa-se que a maioria dos estudos está fortemente concentrada em investigar o comportamento pós-compra de serviços, seguindo a linha mestra dos estudos internacionais. Mais ainda, a maior parte dos estudos se concentrou em dois construtos: qualidade e satisfação. Tipicamente, esses estudos buscam determinar os antecedentes e as conseqüências relacionadas aos dois construtos, utilizando modelagem de equações estruturais. Embora haja razoável dispersão geográfica de estudos realizados por pesquisadores de várias instituições, a maior freqüência de trabalhos nessa linha provém da UFRGS, da UFPE e da UFMG, além de contribuições da UFRJ, UFPR, PUC-RS, FGV-EAESP, USP e Unisinos.

As demais linhas de pesquisa identificadas receberam atenção restrita dos pesquisadores brasileiros. As explorações teóricas e empíricas em torno do tema de cenários de serviços se concentram nos trabalhos realizados na PUC-Rio. Por sua vez, o estudo do processo de escolha do consumidor de serviços também se limitou a poucos estudos e centros de investigação, com ênfase maior nos trabalhos provenientes da UFPE. Finalmente, as poucas investigações sobre estratégia de marketing de serviços têm participação de pesquisadores da UFRGS e PUC-Minas.

Há clara preferência pela perspectiva hipotético-dedutiva, pelos métodos quantitativos de análise e, entre esses, pela modelagem de equações estruturais. Uma exceção são os estudos realizados sobre cenários de serviços, em que se privilegiam a perspectiva interpretativa e métodos qualitativos de análise.

A maior parte dos estudos realizados em marketing de serviços no Brasil pode ser caracterizada como testes empíricos que utilizam instrumentos de medida desenvolvidos por pesquisadores de outros países, tais como a escala Servqual (por exemplo, Dutra, Oliveira e Gouveia, 2002; Rocha e Oliveira, 2003) e os índices ACSI American Customer Satisfaction Index (Gonçalves Filho, Guerra e Moura, 2003; Moura, 2005) e ESCI - European Customer Satisfaction Index (por exemplo, Leite, Elias e Sundermann, 2005), além de operacionalizações de construtos específicos. No entanto, verificam-se cuidados na adequação dessas medidas à realidade brasileira, assim como alguns esforços inovadores de desenvolvimento de modelos teóricos e medidas (por exemplo, Marchetti e Prado, 2001, 2004; Rodrigues, 2000a), contribuições metodológicas (Rosa e Kamakura, 2001) e contribuições ao aperfeiçoamento de modelos existentes (Carvalho e Leite, 1999a, 1999b).

Em síntese, considerando-se como base os trabalhos apresentados no EnAnpad nos últimos sete anos, podese concluir que o tema de marketing de serviços tem sido objeto de investigação de diversos pesquisadores da área de Marketing no país, difundindo-se nas principais instituições de pós-graduação. Seria desejável, porém, uma ampliação do foco atualmente existente em medições de qualidade e satisfação, seus antecedentes e conseqüências, buscando-se explorar novas questões comportamentais e estratégicas relacionadas a serviços.

\section{TENDÊNCIAS}

$\mathrm{Na}$ opinião de diversos autores, as fronteiras de investigação no marketing de serviços continuam a se expandir. Cronin (2003), por exemplo, sugere como temas de investigação a busca de melhor entendimento do processo decisório do consumidor com relação a serviços, assim como um exame do valor adicionado pela oferta de serviços agregados a produtos. Em particular, o autor sugere que a literatura de marketing de serviços está excessivamente centrada na noção de que as percepções que o consumidor tem da qualidade dos serviços levam à percepção de valor e à satisfação. Esta conduziria à intenção de compra, clamando pelo uso de modelos de comportamento do consumidor já existentes na literatura para obter melhor entendimento da ligação entre qualidade percebida e satisfação. Outro tema a ser investigado seria o impacto do ambiente de serviços nas decisões de compra do consumidor. Finalmente, Cronin sugere uma inversão do enfoque adotado, pesquisando-se mais os aspectos negativos do comportamento do consumidor que podem afetar sua decisão de compra: a resistência a comprar, o arrependimento, a insatisfação, a rejeição e a ira do consumidor.

Outros temas que têm atraído interesse recente são o marketing de serviços públicos (Kooiman, 1996), o marketing de serviços e coeficientes (Van der Zwan e Bhamra, 2003), o marketing internacional de serviços (Knight, 1999) e as dimensões éticas dos serviços, particularmente dos serviços profissionais (Rao e Singhapakdi, 1997). Seth, Deshmukh e Vrat (2005) propõem, como um dos temas a serem estudados, o papel da tecnologia, em particular a tecnologia da informação, no marketing de serviços. Entre as questões de pesquisa sugeridas, estão: que tipo de arquitetura de sistemas de informação permitiria maior qualidade de serviços? E como ouvir 
o cliente por meio da tecnologia da informação? Laing et al. (2002) também apontam o papel fundamental da tecnologia da informação em influenciar a forma como se dará a interação com os clientes no futuro, mudando a configuração dos cenários de serviços e levantando a questão da despersonalização dos serviços.

Uma agenda de pesquisas em marketing de serviços no Brasil deveria necessariamente considerar as diferenças sociais e econômicas "que tornam a evolução da gestão de serviços uma tarefa muito mais complexa na América Latina" (Reynoso, 1999, p. 403). De fato, a cultura brasileira é a grande ausente nos estudos de marketing de serviços no país. Qual o impacto de aspectos culturais específicos do Brasil sobre o gerenciamento de serviços, como, por exemplo, o jeitinho brasileiro, ou a dualidade da "casa" e da "rua", de Roberto DaMatta? A cordialidade do brasileiro teria impacto positivo sobre a qualidade dos serviços? As diferenças regionais impactariam a satisfação dos clientes? E de que modo a hierarquia e as relações verticais interferem no processo de entrega de serviços e na resposta dos clientes? O trabalho realizado por Chauvel (2000a, 2000b), ainda que não devotado a serviços, proporciona uma base para a formulação de hipóteses sobre as relações entre cultura e satisfação ou insatisfação do consumidor brasileiro.

As questões sociais, em particular o impacto da desigualdade social, praticamente inexistem nos estudos brasileiros. Uma notável exceção é o trabalho de Carvalho, Faria e Carvalho (2002) sobre a maneira como a baixa escolaridade de operadores de serviços afeta o desempenho de marketing das empresas na visão de seus gerentes. Sugestivamente, o artigo tem como subtítulo "aprendendo a reconhecer o que vem sendo ignorado". Questão correlata é como a baixa qualidade de vida das camadas mais pobres da população brasileira, da qual se originam os operadores de serviços, pode afetar a prestação de serviços de qualidade pelas empresas.

O estudo das relações entre estratégias de marketing de serviços e desempenho merece maior atenção, tendose limitado aos trabalhos de Trez e Luce (2000, 2001). Seth, Deshmukh e Vrat (2005) indicam ser a ligação entre qualidade de serviços e desempenho organizacional uma das questões críticas a merecerem a atenção dos pesquisadores em futuro próximo. Um painel de especialistas em marketing de serviços também indicou a necessidade de dar maior atenção aos resultados da qualidade de serviços: lucratividade, participação no mercado e retenção de clientes (Grove, Fisk e John, 2003). Finalmente, a análise de grupos estratégicos nos setores de serviços, de suas estratégias de marketing e desempenho tem sido ignorada, embora tenha recebido a atenção dos pesquisadores de estratégia empresarial.

\section{REFERÊNCIAS}

ALMEIDA, A. R. D.; BOTELHO, D. Confiança, qualidade ou valor percebido: o que influencia a satisfação na compra de um imóvel? In: ENCONTRO NACIONAL DA ASSOCIAČ̃̃O NACIONAL DOS PROGRAMAS DE PÓS-GRADUAÇÃO E PESQUISA EM ADMINISTRAÇÃO, 29., 2005, Brasília. Anais. Brasília: Anpad, 2005.

ANDRADE, V. M. Gerenciamento de impressões em serviços de hospitalidade: investigando o servicescape em hotéis com base na metáfora teatral In: ENCONTRO NACIONAL DA ASSOCIAÇÃO NACIONAL DOS PROGRAMAS DE PÓS-GRADUAÇÃO E PESQUISA EM ADMINISTRAÇÃO, 28., 2004, Curitiba. Anais. Curitiba: EnAnpad, 2004.

ASUBONTENG, P.; MCCLEARLY, K. J.; SWAN, J. E. SERVQUAL revisited: a critical review of service quality. Journal of Services Marketing, v. 10, n. 6, p. 62-81, 1996.

BRASIL, V. S. Sistemas de prestação de serviços: um enfoque no processo de escolha do consumidor. In: ENCONTRO NACIONAL DA ASSOCIAÇÃO NACIONAL DOS PROGRAMAS DE PÓS-GRADUAÇÃO E PESQUISA EM ADMINISTRAÇÃO, 26., 2002, Salvador. Anais. Salvador: EnAnpad, 2002.

BREI, V. A. Construção de confiança em trocas relacionais de serviço: uma pesquisa nacional com usuários da Internet. In: ENCONTRO NACIONAL DA ASSOCIAÇÃO NACIONAL DOS PROGRAMAS DE PÓS-GRADUAÇÃO E PESQUISA EM ADMINISTRAÇÃO, 27., 2003, Atibaia. Anais. Atibaia EnAnpad, 2003.

BREI, V. A.; Rossi, C. A. V. Confiança, valor percebido e lealdade em trocas relacionais de serviços: um estudo com usuários de internet banking no Brasil. In: ENCONTRO NACIONAL DA ASSOCIAÇÃO NACIONAL DOS PROGRAMAS DE PÓS-GRADUAÇÃO E PESQUISA EM ADMINISTRAÇÃO, 26., 2002, Salvador. Anais. Salvador: EnAnpad, 2002.

BREI, V. A.; ROSSI, C. A. Confiança, valor percebido e lealdade em trocas relacionais de serviço: um estudo com usuários de internet banking no Brasil. Revista de Administração Contemporânea, v. 9, n. 2, p. 145-168, 2005.

BROWN, S. W.; FISK, R. P.; BITNER, M. J. The development and emergence of services marketing thought. International Journal of Service Industry Management, v. 5, n. 1, p. 21-48, 1994.

BUSS, C. O.; BREI, V. A.; GRINBERG, C. S.; ALMEIDA, S.; FREITAS, A. Importância dos serviços ao cliente na compra de aparelhos condicionadores de ar no Estado do Rio Grande do Sul. In: ENCONTRO NACIONAL DA 
ASSOCIAÇÃO NACIONAL DOS PROGRAMAS DE PÓS-GRADUAÇÃO E PESQUISA EM ADMINISTRAÇÃO, 24., 2000, Costão do Santinho. Anais. Costão do Santinho: EnAnpad, 2000.

CARLZON, J. A hora da verdade (Moments of Truth). Rio de Janeiro: COP Editora, 1990.

CARVALHO, F. A.; LEITE, V. F. Refinando a conjetura PBZ: uma revisão da relação entre importância e tolerância em qualidade de serviços. In: ENCONTRO NACIONAL DA ASSOCIAÇÃO NACIONAL DOS PROGRAMAS DE PÓS-GRADUAÇÃO E PESQUISA EM ADMINISTRAÇÃO, 23., 1999a, Foz do Iguaçu. Anais. Foz do Iguaçu: EnAnpad, 1999a.

CARVALHO, F. A.; LEITE, V. F. Attribute importance in service quality: an empirical test of the PBZ conjecture in Brazil. International Journal of Service Industry Management, v. 10, n. 5, p. 487-504, 1999b.

CARVALHO, J. L. F. S.; FARIA, A.; CARVALHO, F. A. Educação e desempenho de marketing de empresas de serviços: aprendendo a reconhecer o que vem sendo ignorado. In: ENCONTRO NACIONAL DA ASSOCIAÇÃO NACIONAL DOS PROGRAMAS DE PÓS-GRADUAČ̃̃O E PESQUISA EM ADMINISTRAÇÃO, 26., 2002, Salvador. Anais. Salvador: Anpad, 2002.

CARVALHO, J. L. F. S.; HEMAIS, M. W.; MOTTA, P. C. Do zen ao techno: as tribos de consumidores e a música nos cenários de serviços. In: ENCONTRO NACIONAL DA ASSOCIAÇÃO NACIONAL DOS PROGRAMAS DE PÓS-GRADUAÇÃO E PESQUISA EM ADMINISTRAÇÃO, 25., 2001, Campinas. Anais. Campinas: Anpad, 2001.

CARVALHO, J. L. F. S.; LIMA, T. R.; MOTTA, P. C. As experiências interativas sociotécnicas com a iluminação de cenários de serviços. In: ENCONTRO NACIONAL DA ASSOCIAÇÃO NACIONAL DOS PROGRAMAS DE PÓS-GRADUAÇÃO E PESQUISA EM ADMINISTRAÇÃO, 27., 2003, Atibaia. Anais. Atibaia: EnAnpad, 2003.

CARVALHO, J. L. F. S.; MOTTA, P. C. Iluminando cenários de serviços: um exame das funções da luz nas "horas da verdade". In: ENCONTRO NACIONAL DA ASSOCIAÇ̃̃O NACIONAL DOS PROGRAMAS DE PÓSGRADUAÇÃO E PESQUISA EM ADMINISTRAÇÃO, 24., 2000, Costão do Santinho. Anais. Costão do Santinho: EnAnpad, 2000.

CARVALHO, J. L. F. S; VERGARA, S. C. Repensando os roteiros de marketing de serviços: análise crítica da teoria dos scripts cognitivos. In: ENCONTRO NACIONAL DA ASSOCIAÇÃO NACIONAL DOS PROGRAMAS DE PÓSGRADUAÇÃO E PESQUISA EM ADMINISTRAÇÃO, 24., 2000, Costão do Santinho. Anais. Costão do Santinho: EnAnpad, 2000.

CHAUVEL, M. A. Insatisfação e queixa à empresa: investigando os relatos dos consumidores. In: ENCONTRO NACIONAL DA ASSOCIAÇÃO NACIONAL DOS PROGRAMAS DE PÓS-GRADUAÇÃO E PESQUISA EM ADMINISTRAÇÃO, 24., 2000a, Costão do Santinho. Anais. Costão do Santinho: EnAnpad, 2000a.

CHAUVEL, M. A. Consumidores insatisfeitos: uma oportunidade para as empresas. Rio de Janeiro: Mauad, 2000b.
CORTIMIGLIA, M. N.; MACADAR, B. M.; DINATO, M. R. O impacto do tipo de relacionamento na lealdade do consumidor em um contexto de falhas de serviço: um estudo experimental no Brasil. In: ENCONTRO NACIONAL DA ASSOCIAÇÃO NACIONAL DOS PROGRAMAS DE PÓSGRADUAČ̃̃ E PESQUISA EM ADMINISTRAÇÃO, 27., 2003, Atibaia. Anais. Atibaia: EnAnpad, 2003.

CRONIN JR., J. J. Looking back to see forward in services marketing: some ideas to consider. Managing Service Quality, v. 13, n. 5, p. 332-337, 2003.

DE TONI, D.; MILAN, G. S.; BARAZETTI, L. Imagens de serviços: um estudo exploratório sobre a configuração e organização das imagens dos serviços de fisioterapia de um plano de saúde. In: ENCONTRO NACIONAL DA ASSOCIAÇÃO NACIONAL DOS PROGRAMAS DE PÓS-GRADUAÇÃO E PESQUISA EM ADMINISTRAÇÃO, 28., 2004, Curitiba. Anais. Curitiba: Anpad, 2004

DE TONI, D.; MILAN, G. S.; SCHULER, M. Configuração de imagens de serviços : um estudo aplicado aos serviços de fisioterapia disponibilizados por um plano de saúde. In: ENCONTRO NACIONAL DA ASSOCIAÇÃO NACIONAL DOS PROGRAMAS DE PÓS-GRADUAÇÃO E PESQUISA EM ADMINISTRAÇÃO, 29., 2005, Brasília. Anais. Brasília: Anpad, 2005.

DUTRA, H. F. O.; OLIVEIRA, P. A. S.; GOUVEIA, T. B. Avaliando a qualidade de serviço numa instituição de ensino superior. In: ENCONTRO NACIONAL DA ASSOCIAÇÃO NACIONAL DOS PROGRAMAS DE PÓSGRADUAÇÃO E PESQUISA EM ADMINISTRAÇÃO, 27., 2002, Salvador Anais. Salvador: Anpad, 2000.

FARIAS, S. A.; GÓIS, G. T.; OLIVEIRA, L. A. C. Por que isto ocorreu? Utilizando a teoria de atribuição para explicar as saídas no consumo de serviços. In: ENCONTRO NACIONAL DA ASSOCIACCÃO NACIONAL DOS PROGRAMAS DE PÓS-GRADUAÇÃO E PESQUISA EM ADMINISTRAÇÃO, 24., 2000, Costão do Santinho. Anais. Costão do Santinho: EnAnpad, 2000.

FARIAS, S. A.; SANTOS, R. C. Influência da resposta afetiva na satisfação do consumidor: um estudo no segmento da terceira idade. In: ENCONTRO NACIONAL DA ASSOCIAÇÃO NACIONAL DOS PROGRAMAS DE PÓSGRADUAÇÃO E PESQUISA EM ADMINISTRAÇÃO, 24., 2000, Costão do Santinho. Anais. Costão do Santinho: Anpad, 2000.

FONSECA, F. R. B.; NETO, A. F. S.; CORDEIRO, A. T.; GOUVEIA, T. B. Risco percebido e estratégias redutoras de risco através de diferentes tipos de serviços. In: ENCONTRO NACIONAL DA ASSOCIAÇÃO NACIONAL DOS PROGRAMAS DE PÓS-GRADUAÇÃO E PESQUISA EM ADMINISTRAÇÃO, 26., 2002, Salvador. Anais. Salvador: Anpad, 2002.

FONSECA, M. J.; TREZ, G. O impacto das falhas dos serviços e ações de recuperação na satisfação, lealdade e confiança. In: ENCONTRO NACIONAL DA ASSOCIAČ̃̃O NACIONAL DOS PROGRAMAS DE PÓSGRADUAÇÃO E PESQUISA EM ADMINISTRAÇÃO, 29., 2005, Brasília Anais. Brasília: Anpad, 2005. 
GASTAL, F; LUCE, F. B. A influência da satisfação e dos custos de mudança na lealdade do cliente. In: ENCONTRO NACIONAL DA ASSOCIAÇÃO NACIONAL DOS PROGRAMAS DE PÓS-GRADUAÇÃO E PESQUISA EM ADMINISTRAÇÃO, 29., 2005, Brasília. Anais. Brasília: Anpad, 2005.

GHISI, M. A.; MERLO, E. M.; NAGANO, M. S. A mensuração da importância de atributos em serviços: uma comparação de escalas. In: ENCONTRO NACIONAL DA ASSOCIAÇÃO NACIONAL DOS PROGRAMAS DE PÓSGRADUAÇÃO E PESQUISA EM ADMINISTRAÇÃO, 28., 2004, Curitiba. Anais. Curitiba: Anpad, 2004.

GONÇALVES FILHO, C.; GUERRA, R. S.; MOURA, A. Mensuração de satisfação, qualidade, lealdade, valor expectativa em instituições de ensino superior: um estudo do modelo ASCI através de equações estruturais. In: ENCONTRO NACIONAL DA ASSOCIAÇÃO NACIONAL DOS PROGRAMAS DE PÓS-GRADUAÇÃO E PESQUISA EM ADMINISTRAÇÃO, 27., 2003, Atibaia. Anais. Atibaia: EnAnpad, 2003.

GRÖNROOS, C. Strategic Management and Marketing in the Service Sector. Cambridge, MA: Marketing Science Institute, 1983

GRÖNROOS, C. A service quality model and its marketing implications. European Journal of Marketing, v. 18, n. 4, p. 36-44, 1984.

GRÖNROOS, C. Marketing: gerenciamento e serviços. Rio de Janeiro: Campus, 1995.

GROVE, S. J.; FISK, R. P.; JOHN, J. The future of services marketing: forecasts from ten service experts. Journal of Services Marketing, v. 17, n. 2, p. 107-121, 2003.

KNIGHT, G. International services marketing: review of research 19801998. Journal of Services Marketing, v. 13, n. 4/5, p. 347-360, 1999.

KOOIMAN, J. Research and theory about new public services management: review and agenda for the future. International Journal of Services Industry Management, v. 9, n. 5/6, p. 7-22, 1996.

KOTLER, P. Prólogo. In: GRÖNROOS, C. Marketing: gerenciamento e serviços. Rio de Janeiro: Campus, 1995. p. 9-11.

LAING, A.; LEWIS, B.; FOXALL, G.; HOGG, G. Predicting a diverse future: directions and issues in the marketing of services. European Journal of Marketing, v. 36, n. 4, p. 479-494, 2002.

LARÁN, J. A.; ESPINOZA, F. S. Consumidores satisfeitos, e então? Analisando a satisfação como antecedente da lealdade. Revista de Administração Contemporânea, v. 8, n. 2, p. 51-70, 2004.

LEITE, R. S.; ELIAS, C. L.; SUNDERMANN, J. O índice europeu de satisfação de clientes (ECSI): um estudo empírico de sua aplicação em uma empresa brasileira fornecedora de ERP. In: ENCONTRO NACIONAL DA ASSOCIAÇÃO NACIONAL DOS PROGRAMAS DE PÓS-GRADUAÇÃO E PESQUISA EM ADMINISTRAÇÃO, 29., 2005, Brasília. Anais. Brasília: Anpad, 2005.
LEITE, V. F; CARVALHO, F. A. As expectativas dos heavy users de serviços são distintas daquelas dos usuários menos freqüentes? In: ENCONTRO NACIONAL DA ASSOCIAÇÃO NACIONAL DOS PROGRAMAS DE PÓSGRADUAÇÃO E PESQUISA EM ADMINISTRAÇÃO, 24., 2000, Costão do Santinho. Anais. Costão do Santinho: Anpad, 2000.

LOVELOCK, C. H. Classifying services to gain strategic marketing insights. Journal of Marketing, v. 47, n. 3, p. 9-20, 1983.

LOVELOCK, C. H. Services Marketing: Text, Cases, and Readings. Englewood Cliffs, NJ: Prentice-Hall, 1984.

MACHADO, F. B. Um estudo sobre os níveis de fidelidade do consumidor em serviços de naturezas distintas. In: ENCONTRO NACIONAL DA ASSOCIAÇÃO NACIONAL DOS PROGRAMAS DE PÓS-GRADUAÇÃO E PESQUISA EM ADMINISTRAÇÃO, 28., 2004, Curitiba. Anais. Curitiba: Anpad, 2004.

MARCHETTI, R.; PRADO, P. Avaliação da satisfação do consumidor utilizando o PLS: um modelo aplicado ao setor elétrico brasileiro. In: ENCONTRO NACIONAL DA ASSOCIAÇÃO NACIONAL DOS PROGRAMAS DE PÓS-GRADUAÇÃO E PESQUISA EM ADMINISTRAÇÃO, 25., 2001, Campinas. Anais. Campinas: Anpad, 2001.

MARCHETTI, R.; PRADO, P. H. M. Avaliação da satisfação do consumidor utilizando o método de equações estruturais: um modelo aplicado ao setor elétrico brasileiro. Revista de Administração Contemporânea, v. 8, n. 4, p. 9-32, 2004.

MARTIN, C. L. The history, evolution and principles of service marketing: poised for the new millennium. Marketing Intelligence \& Planning, v. 17 , n. 7, p. 324-328, 1999.

MOORI, R. G.; MARCONDES, R. C.; ÁVILA, R. T. A análise de agrupamentos como instrumento de apoio à melhoria da qualidade dos serviços aos clientes. Revista de Administração Contemporânea, v. 6, n. 1, p. 63-84, 2002.

MOURA, A. C. Validação do modelo de satisfação ACSI modificado no setor de telefonia móvel. In: ENCONTRO NACIONAL DA ASSOCIAÇÃO NACIONAL DOS PROGRAMAS DE PÓS-GRADUAÇÃO E PESQUISA EM ADMINISTRAÇÃO, 29., 2005, Brasília. Anais. Brasília: Anpad, 2005.

NETO, A. F. S.; GOUVEIA, T. B.; CORDEIRO, A. T.; FONSECA, F. R. B. Tamanho do conjunto de consideração: um estudo comparativo entre três categorias de serviços. In: ENCONTRO NACIONAL DA ASSOCIAÇÃO NACIONAL DOS PROGRAMAS DE PÓS-GRADUAČ̃̃O E PESQUISA EM ADMINISTRAÇÃO, 26., 2002, Salvador. Anais. Salvador: Anpad, 2002.

NETO, A. F. S. Compreendendo o processo de escolha do consumidor utilizando dois modelos cognitivos: um estudo comparativo no setor de serviços. In: ENCONTRO NACIONAL DA ASSOCIAÇÃO NACIONAL DOS PROGRAMAS DE PÓS-GRADUAÇÃO E PESQUISA EM ADMINISTRAÇÃO, 27., 2003, Atibaia. Anais. Atibaia: EnAnpad, 2003. 
NETO, J. C. Os serviços ao cliente como diferencial competitivo: implicações para a estrutura e a gerência das organizações. In: ENCONTRO NACIONAL DA ASSOCIAÇÃO NACIONAL DOS PROGRAMAS DE PÓSGRADUAÇÃO E PESQUISA EM ADMINISTRAÇÃO, 24., 2000, Costão do Santinho. Anais. Costão do Santinho: Anpad, 2000.

NORMANN, R. Management for Growth. Chichester: Wiley, 1977.

NORMANN, R. Administração de serviços: estratégia e liderança na empresa de serviços. São Paulo: Atlas, 1993.

OLIVEIRA, P. A. S.; DUTRA, H. F. O. Percepção de qualidade e construção de relacionamentos: uma investigação num setor financeiro. In: ENCONTRO NACIONAL DA ASSOCIAÇÃO NACIONAL DOS PROGRAMAS DE PÓS-GRADUAÇÃO E PESQUISA EM ADMINISTRAÇÃO, 26., 2002, Salvador. Anais. Salvador: Anpad, 2002.

PARASURAMAN, A; BERRY, L. L.; ZEITHAML, V. A. Refinement and reassessment of the Servqual scale. Journal of Retailing, v. 67, n. 4, p. 420-450, Winter 1991.

PARASURAMAN, A.; ZEITHAML, V. A.; BERRY, L. L. A conceptual model of service quality and its implications for future research. Journal of Marketing, v. 49, n. 4, p. 41-50, Fall 1985.

PARASURAMAN, A.; ZEITHAML, V. A.; BERRY, L. L. Servqual: a multiple item scale for measuring consumer perception of service quality. Journal of Retailing, v. 64, n. 1, p. 12-37, 1988.

PARASURAMAN, A.; ZEITHAML, V. A.; BERRY, L. L. Reassessment of expectations as a comparison standard in measuring service quality: implications for future research. Journal of Marketing, v. 58, n. 1, p. 111124,1994

PRADO, P. H. M. Integração da qualidade percebida, resposta afetiva e satisfação no processo de compra-consumo de serviços. In: ENCONTRO NACIONAL DA ASSOCIAÇÃO NACIONAL DOS PROGRAMAS DE PÓSGRADUAÇÃO E PESQUISA EM ADMINISTRAÇÃO, 26., 2002, Salvador. Anais. Salvador: Anpad, 2002.

RAO, C. P.; SINGHAPAKDI, S. Marketing ethics: a comparison between services and other marketing professionals. Journal of Services Marketing, v. 11, n. 6, 1997.

RÉVILLION, A. S. P.; ROSSI, C. A. V. Supermercados pequenos, médios e grandes: um estudo sobre a satisfação do consumidor com o setor supermercadista em Porto Alegre. In: ENCONTRO NACIONAL DA ASSOCIAÇÃO NACIONAL DOS PROGRAMAS DE PÓS-GRADUAÇÃO E PESQUISA EM ADMINISTRAÇÃO, 24., 2000, Costão do Santinho. Anais. Costão do Santinho: Anpad, 2000.

REYNOSO, J. Progress and prospects of service management in Latin America. International Journal of Services Industry Management, v. 10, n. 5, p. 401-408, 1999.
ROCHA, V. A. G. A.; OLIVEIRA, P. A. S. Avaliando a qualidade de serviço: aplicação da escala Servqual numa grande instituição bancária. In: ENCONTRO NACIONAL DA ASSOCIAÇÃO NACIONAL DOS PROGRAMAS DE PÓS-GRADUAÇÃO E PESQUISA EM ADMINISTRAÇÃO, 27., 2003, Atibaia. Anais. Atibaia: EnAnpad, 2003.

RODRIGUES, A. C. M. Uma escala de mensuração da zona de tolerância de consumidores de serviços. In: ENCONTRO NACIONAL DA ASSOCIAÇÃO NACIONAL DOS PROGRAMAS DE PÓS-GRADUAÇÃO E PESQUISA EM ADMINISTRAÇÃO, 24., 2000a, Costão do Santinho. Anais. Costão do Santinho: Anpad, 2000a.

RODRIGUES, A. C. M. Qualidade de serviços e satisfação do consumidor: aspectos conceituais distintos. In: ENCONTRO NACIONAL DA ASSOCIAÇÃO NACIONAL DOS PROGRAMAS DE PÓS-GRADUAÇÃO E PESQUISA EM ADMINISTRAÇÃO, 24., 2000b, Costão do Santinho. Anais. Costão do Santinho: Anpad, 2000b.

RODRIGUES, J. M. S. Gestão da satisfação e da fidelidade de consumidores: um estudo dos fatores que afetam a satisfação e a fidelidade no mercado de turismo. In: ENCONTRO NACIONAL DA ASSOCIAÇÃO NACIONAL DOS PROGRAMAS DE PÓS-GRADUAÇÃO E PESQUISA EM ADMINISTRAÇÃO, 28., 2004, Curitiba. Anais. Curitiba: Anpad, 2004.

ROSA, F; KAMAKURA, W. A. Pesquisas de satisfação de clientes e efeito halo: interpretações equivocadas? In: ENCONTRO NACIONAL DA ASSOCIAÇÃO NACIONAL DOS PROGRAMAS DE PÓS-GRADUAÇÃO E PESQUiSA EM ADMINISTRAÇÃO, 25., 2001, Campinas. Anais. Campinas: Anpad, 2001.

ROSSI, C. A. V.; LUCE, F. B.; MAZZON, J. F; SLONGO, L. A. Solução clássica para um problema contemporâneo: a relevância da satisfação de clientes para segmentar mercados na telefonia celular brasileira. In: ENCONTRO NACIONAL DA ASSOCIAČ̃̃O NACIONAL DOS PROGRAMAS DE PÓS-GRADUAÇÃO E PESQUISA EM ADMINISTRAÇÃO, 28., 2004, Curitiba. Anais. Curitiba: Anpad, 2004.

SANTOS, C. P. Construção e teste de um modelo teórico sobre o impacto do gerenciamento de reclamações na confiança e lealdade do consumidor, no contexto de troca de serviços relacionais. In: ENCONTRO NACIONAL DA ASSOCIAÇÃO NACIONAL DOS PROGRAMAS DE PÓS-GRADUAÇÃO E PESQUISA EM ADMINISTRAÇÃO, 25., 2001, Campinas. Anais. Campinas: Anpad, 2001.

SANTOS, C. P.; FERNANDES, D. H. A recuperação de serviços como ferramenta de relacionamento: seu impacto na confiança e lealdade dos clientes. In: ENCONTRO NACIONAL DA ASSOCIAÇÃO NACIONAL DOS PROGRAMAS DE PÓS-GRADUAÇÃO E PESQUISA EM ADMINISTRAÇÃO, 29., 2005, Brasília. Anais. Brasília: Anpad, 2005.

SANTOS, L. C.; MUNIZ, R. M. M. Qualicredicom: uma escala operacional pioneira para a mensuração da qualidade da entrega de serviços de crédito bancário empresarial no Brasil. In: ENCONTRO NACIONAL DA ASSOCIAÇÃO NACIONAL DOS PROGRAMAS DE PÓS-GRADUAÇÃO 
E PESQUISA EM ADMINISTRAÇÃO, 28., 2004, Curitiba. Anais. Curitiba: Anpad, 2004.

SANTOS, C. P.; ROSSI, C. A. V. Os antecedentes da confiança do consumidor em episódios envolvendo reclamações sobre serviços. In: ENCONTRO NACIONAL DA ASSOCIAÇÃO NACIONAL DOS PROGRAMAS DE PÓSGRADUAÇÃO E PESQUISA EM ADMINISTRAÇÃO, 26., 2002, Salvador. Anais. Salvador: Anpad, 2002

SETH, N.; DESHMUHK, S. G.; VRAT, P. Service quality models: a review. International Journal of Quality and Reliability Management, v. 22, n. 9, p. 913-949, 2005.

SHOSTACK, G. L. Breaking free from product marketing. Journal of Marketing, v. 41, n. 2, p. 73-80, Apr. 1977.

SLONGO, L. A.; MÜSSNICH, R. Serviços ao cliente e marketing de relacionamento no setor hoteleiro de Porto Alegre. Revista de Administração Contemporânea, v. 9, n. 1, p. 149-170, 2005.

TREZ, G.; LUCE, F. B. Os serviços ao cliente como estratégia de marketing. In: ENCONTRO NACIONAL DA ASSOCIAÇÃO NACIONAL DOS PROGRAMAS DE PÓS-GRADUAÇÃO E PESQUISA EM ADMINISTRAÇÃO, 24., 2000, Costão do Santinho. Anais. Costão do Santinho: Anpad, 2000.

TREZ, G.; LUCE, F. B. Os serviços ao cliente e a performance da empresa. In: ENCONTRO NACIONAL DA ASSOCIAÇÃO NACIONAL DOS PROGRAMAS DE PÓS-GRADUAÇÃO E PESQUISA EM ADMINISTRAÇÃO, 25., 2001, Campinas. Anais. Campinas: Anpad, 2001.
URDAN, A. T.; HUERTAS, M. K. Z. O médico e a qualidade percebida pelo paciente. In: ENCONTRO NACIONAL DA ASSOCIAÇÃO NACIONAL DOS PROGRAMAS DE PÓS-GRADUAÇÃO E PESQUISA EM ADMINISTRAÇÃO, 27., 2003, Atibaia. Anais. Atibaia: EnAnpad, 2003.

VAN DER ZWAN, F; BHAMRA, T. Services management: taking up the sustainable development challenge. Journal of Services Marketing, v. 17, n. 4, p. 341-356, 2003.

VECCHI, A. J. S.; LUCE, F. B. As conseqüências comportamentais da qualidade em serviços: um caso no B2B. In: ENCONTRO NACIONAL DA ASSOCIAÇÃO NACIONAL DOS PROGRAMAS DE PÓS-GRADUAÇÃO E PESQUISA EM ADMINISTRAÇÃO, 25., 2001, Campinas. Anais. Campinas: Anpad, 2001.

VEIGA, R. T.; GONÇALVES, C. A.; MOURA, A. I. Um exame empírico do modelo de conseqüências comportamentais da qualidade de serviços. In: ENCONTRO NACIONAL DA ASSOCIAÇÃO NACIONAL DOS PROGRAMAS DE PÓS-GRADUAÇÃO E PESQUISA EM ADMINISTRAÇÃO, 26., 2002, Salvador. Anais. Salvador: Anpad, 2002.

ZUÑIGA, M. K. H.; URDAN, A. T. Satisfação dos clientes com serviços de assistência técnica automobilística e lealdade dele ao fabricante do veículo. In: ENCONTRO NACIONAL DA ASSOCIAÇÃO NACIONAL DOS PROGRAMAS DE PÓS-GRADUAÇÃO E PESQUISA EM ADMINISTRAÇÃO, 24., 2000, Costão do Santinho. Anais. Costão do Santinho: Anpad, 2000.

\section{Artigo convidado. Aprovado em 31.08.2006.}

